\title{
Vacuum ultraviolet photolysis of hydrogenated amorphous carbons
}

\section{Interstellar $\mathrm{H}_{2}$ and $\mathrm{CH}_{4}$ formation rates}

\author{
I. Alata ${ }^{1,2, \star}$, G. A. Cruz-Diaz ${ }^{3}$, G. M. Muñoz Caro ${ }^{3}$, and E. Dartois ${ }^{1,2}$ \\ ${ }^{1}$ CNRS-INSU, Institut d'Astrophysique Spatiale, UMR 8617, 91405 Orsay, France \\ e-mail: ialata@ias.u-psud.fr \\ 2 Université Paris Sud, Institut d'Astrophysique Spatiale, UMR 8617, Bâtiment 121, 91405 Orsay, France \\ ${ }^{3}$ Centro de Astrobiología, INTA-CSIC, Carretera de Ajalvir, km 4, Torrejón de Ardoz, 28850 Madrid, Spain
}

Received 22 November 2013 / Accepted 9 July 2014

\begin{abstract}
Context. The interstellar hydrogenated amorphous carbons (HAC or a-C:H) observed in the diffuse medium are expected to disappear in a few million years, according to the destruction time scale from laboratory measurements. The existence of a-C:H results from the equilibrium between photodesorption, radiolysis, hydrogenation and resilience of the carbonaceous network. During this processing, many species are therefore injected into the gas phase, in particular $\mathrm{H}_{2}$, but also small organic molecules, radicals or fragments.

Aims. We perform experiments on interstellar a-C:H analogs to quantify the release of these species in the interstellar medium.

Methods. The vacuum ultraviolet (VUV) photolysis of interstellar hydrogenated amorphous carbon analogs was performed at low $(10 \mathrm{~K})$ to ambient temperature, coupled to mass-spectrometry detection and temperature-programed desorption. Using deuterium isotopic substitution, the species produced were unambiguously separated from background contributions.

Results. The VUV photolysis of hydrogenated amorphous carbons leads to the efficient production of $\mathrm{H}_{2}$ molecules, but also to small hydrocarbons.

Conclusions. These species are formed predominantly in the bulk of the a-C:H analog carbonaceous network, in addition to the surface formation. Compared with species made by the recombination of $\mathrm{H}$ atoms and physisorbed on surfaces, they diffuse out at higher temperatures. In addition to the efficient production rate, it provides a significant formation route in environments where the short residence time scale for $\mathrm{H}$ atoms inhibits $\mathrm{H}_{2}$ formation on the surface, such as PDRs. The photolytic bulk production of $\mathrm{H}_{2}$ with carbonaceous hydrogenated amorphous carbon dust grains can provide a very large portion of the contribution to the $\mathrm{H}_{2}$ molecule formation. These dust grains also release small hydrocarbons (such as $\mathrm{CH}_{4}$ ) into the diffuse interstellar medium, which contribute to the formation of small carbonaceous radicals after being dissociated by the UV photons in the considered environment. This extends the interstellar media environments where $\mathrm{H}_{2}$ and small hydrocarbons can be produced.
\end{abstract}

Key words. astrochemistry - molecular processes - ISM: abundances - ultraviolet: ISM - methods: laboratory: solid state dust, extinction

\section{Introduction}

Several carbonaceous solids are observed in the interstellar medium (ISM): nanodiamonds, fullerenes, polyaromatic hydrocarbons, amorphous carbons, hydrogenated amorphous carbons (HACs), and ice mantles that, when processed, can lead to the formation of organic residues. The hydrogenated solids comprise nanodiamonds, observed through vibration bands at 3.43 and $3.53 \mu \mathrm{m}$ of hydrogen-terminated nanodiamonds (Chang et al. 1995; Guillois et al. 1999; Pirali et al. 2007) in equilibrium with the stellar radiation field. They are observed close to the stars around very few objects (Habart et al. 2004; Acke et al. 2006; Goto et al. 2009). The aromatic infrared bands (AIB), with emission bands observed around 3.3, 6.2, 7.7, 8.6, and $11.3 \mu \mathrm{m}$, characteristic of a (hydrogenated) polyaromatic material, are observed ubiquitously in the ISM. They have led to the polycyclic aromatic hydrocarbons (PAHs) hypothesis, which attributes the

\footnotetext{
* This work was supported by the ANR COSMISME project, grant ANR-2010-BLAN-0502 of the French Agence Nationale de la Recherche. Part of the equipment used in this work has been financed by the ANR and French INSU-CNRS program "Physique et Chimie du Milieu Interstellaire" (PCMI).
}

AIB bands to the infrared fluorescence of large PAH molecules upon absorption of energetic UV photons (Leger et al. 1984; Allamandola et al. 1985). The AIB astronomical spectra display a variability in their band profiles, and the observed sources have been classified into three classes $\mathrm{A}-\mathrm{C}$, based on a phenomenological decomposition of the band positions. By number, class $\mathrm{A}$ spectra are the most often observed, followed by class B, and a few class $C$. The evolution among the profiles is primarily linked to differences in the chemical composition of the emitting molecules or particles. Class A are probably the most aromatic ones, and class $\mathrm{C}$ display additional features that are attributed to a more aliphatic character, observationnally (Sloan et al. 2007; Boersma et al. 2008; Keller et al. 2008; Acke et al. 2010), and experimentally (Pino et al. 2008; Carpentier et al. 2012; Gadallah et al. 2013).

Hydrogenated amorphous carbons, also called a-C:H by physicists, for amorphous material made of $\mathrm{C}$ and $\mathrm{H}$, are another component of interstellar dust. In this article we focus on this major phase, which probably hosts the largest carbon portion of these hydrogenated carbon materials. Allen \& Wickramasenghe (1981) observed it for the first time at $3.4 \mu \mathrm{m}$ against a Galactic center source. The features contributing to this absorption band 
Table 1. Characterization of different irradiated a-C:H, a-C:D and polyethylene films.

\begin{tabular}{ccc}
\hline \hline Film & $\begin{array}{c}\text { Thickness } \\
(\mu \mathrm{m})\end{array}$ & $\begin{array}{c}\text { Temperature } \\
(\mathrm{K})\end{array}$ \\
\hline Polyethylene & 15 & 290 \\
a-C:H & 1.4 & 10.2 \\
a-C:H & 4.2 & 10.2 \\
a-C:H & 2.6 & 10.2 \\
a-C:D & 3.4 & 10.2 \\
a-C:D & 3.1 & 10.2 \\
a-C:D & 2.3 & 10.2 \\
\hline
\end{tabular}

were early associated to $\mathrm{sp}^{3} \mathrm{CH}_{3}$ and $\mathrm{CH}_{2}$ stretching modes, as stated for example in Duley \& Williams (1983). Since then, numerous experiments, observations and models have been employed to constrain its origin (e.g., Jones et al. 1983, 2013; Butchart et al. 1986; Mc Fadzean et al. 1989; Ehrenfreund et al. 1991; Sandford et al. 1991, 1995; Pendleton et al. 1994; Tielens et al. 1996; Geballe et al. 1998; Muñoz Caro et al. 2001; Chiar et al. 2002; Mennella et al. 2002; Pendleton \& Allamandola 2002; Dartois et al. 2005; Godard et al. 2012). The a-C:H Galactic abundance has been estimated from the observed $\mathrm{CH}$ stretching modes.

Depending on the intrinsic strength for $\mathrm{CH}$ modes and the degree of hydrogenation of the assumed material carriers, the implied cosmic carbon fraction varies from $2.6 \%$ to $35 \%$ (Sandford et al. 1991), above 2.5 to $4 \%$ based on the spectra of alkanes (Pendleton et al. 1994) and up to 20-30\% for laboratory analogs of a-C:H (Duley 1994; Duley et al. 1998). With the increase in sensitivity studies, a-C:H absorptions have been observed in several extragalactic obscured active galactic nucleus (AGN) sources, mainly via the stretching modes (Pendleton et al. 1994; Mason et al. 2004; Dartois et al. 2004; Imanishi 2006; Risaliti et al. 2006; Imanishi et al. 2008).

Several laboratory analogs have been proposed to account for this carbonaceous component and provide a fit to the observed infrared features. These include analogs produced using a heated carbon rod (Schnaiter et al. 1998), laser-desorbed carbon (Mennella et al. 1999), and subsequent exposure to hydrogen plasma, carbon vapor obtained by striking arc discharges between carbon rods in a hydrogen atmosphere (Mennella et al. 2003), plasma deposition (Lee \& Wdowiak 1993; Furton et al. 1999; Godard \& Dartois 2010), or photoproduced a-C:H at low temperature (Dartois et al. 2005).

These analogs possess commonalities that allow us to define them as a family of carbonaceous materials. In addition to this common infrared active bands, the $\mathrm{H} / \mathrm{C}$ ratio of the above mentioned analogs vary by more than an order of magnitude and also possess intrinsic structural differences that are sometimes much less evident because they imply less active IR modes of the carbonaceous backbone.

$\mathrm{H}_{2}$, the most abundant interstellar molecule, is involved in many of the dominant processes that govern the ISM. However, because of the lack of permanent dipole, coupled to a large rotational spacing (equivalent to about $1000 \mathrm{~K}$ in $T$ ), $\mathrm{H}_{2}$ is often difficult to observe. Astronomical observations of $\mathrm{H}_{2}$ toward relatively energetic media such as photon dominated regions (PDRs), shocks, proto-planetary nebulae (PPNe), or the inner parts, close to stars of young stellar objects (YSOs), suggest that formation of $\mathrm{H}_{2}$ on PAHs or small grains is very frequent in PDRs (e.g., Table 1 of Habart et al. 2005). In parallel, many hydrocarbons species are detected in PDRs, coincident with intense

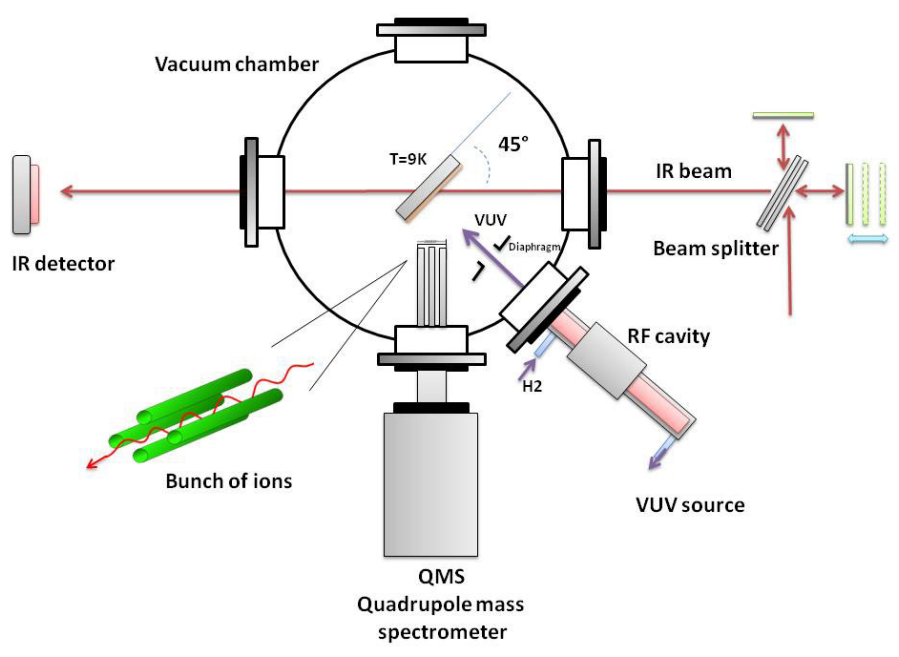

Fig. 1. Schematic view of the SICAL experiment for VUV irradiation, mainly composed of a vacuum chamber containing several windows for FTIR measurement of the solid sample and VUV irradiation beam and quadrupole mass-spectrometer (QMS) for the detection of volatile species in the gas.

emission in the $\mathrm{H}_{2}$ ro-vibrational lines (Pety et al. 2005), following energetic UV excitation. In addition, pure gas-phase chemistry models are unsuccesful in reproducing the measured abundances in the ISM small hydrocarbons. Information on potential sources of these species are thus required to explain the observations. In particular, PAHs are among the targeted precursors to explain the occurrence of small hydrocarbons in PDRs (Pety et al. 2005). Small carbonaceous particles, such as a-C:H, may also play a role in the observed sequence of radicals and neutral species.

In this article we investigate, with experiments that combine infrared and mass spectrometry, the role of energetic UV photons of astrophysical interest that interact with a plasmaproduced HAC, an analog to the a-C:H diffuse ISM dust. We also investigate its deuterated form to disambiguate involved species in mass-spectrometry analyses. The photolysis of this a-C:H (a-C:D) analog releases $\mathrm{H}_{2}\left(\mathrm{D}_{2}\right)$ as the main product, but also some small carbonaceous species that will replenish the gas phase in the diffuse ISM and more energetic environments such as PDRs. In this article we focus on the $\mathrm{H}_{2}$ and $\mathrm{CH}_{4}$ production efficiency. We describe in Sect. 2 the experimental setup and measurements. The results are given in Sect. 3, and the astrophysical implications are discussed in relation to other analogs in Sect. 4, followed by the conclusion in in Sect. 5 .

\section{Experimental details}

\subsection{Experimental setup}

The SICAL-X experimental setup used to study the vacuum ultraviolet (VUV) irradiation of deuterated and HAC (a-C:H, a$\mathrm{C}: \mathrm{D})$ is shown in Fig. 1. It is mainly composed of a vacuum chamber, with a typical working pressure of $\leq 2 \times 10^{-8}$ mbar, obtained with a Pfeiffer 20001/s hybrid turbomolecular pump. Two ports equipped with CsI windows are connected to the chamber, allowing the entry of the IR probe beam. A VUV window $\left(\mathrm{MgF}_{2}\right)$ interfaces the ultraviolet $\mathrm{H}_{2}$ discharge lamp. The HACs, produced in a different setup (SICAL-P) aside from the main experiment, is introduced as a thin film deposited on a $\mathrm{ZnSe}$ substrate, transparent in the mid-IR wavelength range. The substrate is cooled by a closed-cycle helium refrigerator (SRDK series 


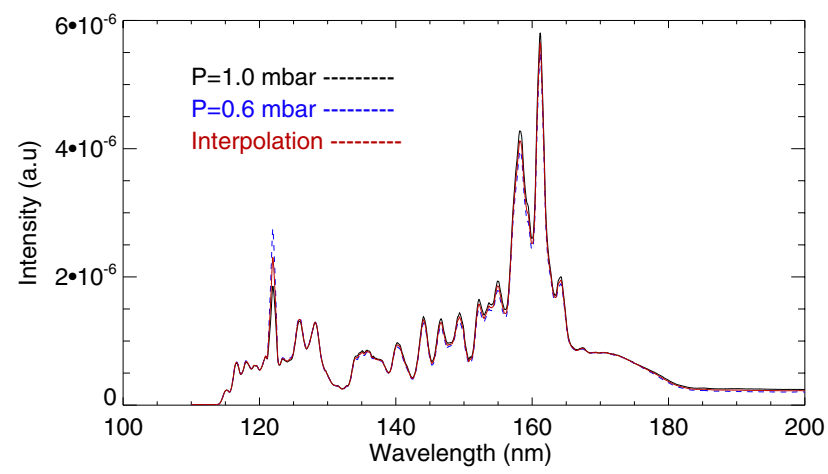

Fig. 2. Emission spectrum of the $\mathrm{H}_{2}$ discharge VUV lamp between 110 and $200 \mathrm{~nm}$ at different pressures, through one $\mathrm{MgF}_{2}$ window, which has an cutoff at $115 \mathrm{~nm}$. The spectra were recorded by Cruz-Diaz et al. 2013 under a hydrogen pressure of 0.6 mbar (blue dashed line) and 1.0 mbar (black continuous line). The expected spectrum of our lamp for 0.75 mbar (red continuous line) is the interpolation of these two curves.

CRYOCOOLER F-50 series Compressor Unit). The temperature of the substrate is adjustable by a resistive-type heater element and monitored with a thermocouple with an accuracy of $\pm 0.1 \mathrm{~K}$. The temperature accessed is about $10 \mathrm{~K}$. The evolution of the film during irradiation is monitored with a Bruker Vertex $80 \mathrm{v}$ infrared Fourier transform spectrometer, at a resolution of $1 \mathrm{~cm}^{-1}$, in a spectral range covering between 7500 and $400 \mathrm{~cm}^{-1}$. During the irradiation many species are liberated from the film surface. We use a QMS quadrupole mass spectrometer (Quadera QMS 200) to follow the evolution of those masses.

\subsection{HAC production}

The hydrogenated and deuterated amorphous carbon films are prepared by a plasma-enhanced chemical vapor deposition method (PECVD), where radicals and ions resulting from a low pressure radio-frequency (RF) plasma (at $2.45 \mathrm{GHZ}$ ), of $\mathrm{CH}_{4}$ or $\mathrm{CD}_{4}$ gases, are deposited on a substrate $\left(\mathrm{ZnSe}, \mathrm{MgF}_{2}\right)$ under vacuum. This method has been described in previous works (Godard et al. 2011; Godard \& Dartois 2010). The typical deposition time required to produce a film several micrometers thick is a few minutes. After the film is produced, the chamber is filled with nitrogen at a pressure slightly higher than the atmosphere and immediately transferred to the high vacuum chamber.

\subsection{VUV irradiation}

VUV photons are generated by a hydrogen flow-discharge lamp, using an Evenson cavity coupled to a $2.45 \mathrm{GHz}$ RF microwave generator. The hydrogen pressure in the lamp was set to 0.75 mbar, which maximises the total number of photons whose energy lies between 6.8 and $10.5 \mathrm{eV}$ (between 120 and $180 \mathrm{~nm})$. The VUV and visible photons enter to the vacuum chamber through a $\mathrm{MgF}_{2}$ window with a cutoff at about $115 \mathrm{~nm}$. The 110 to $200 \mathrm{~nm}$ emission spectra of the VUV lamp used in a similar setup and recorded by Cruz-Diaz et al. (2013) at 0.6 and 1 mbar are presented in Fig. 2, as well as the expected interpolated spectrum with our working $\mathrm{H}_{2}$ lamp pressure ( 0.75 mbar). Using the normalized spectrum and performing an integration over the energy range, the average energy per emitted photon is $\left\langle E_{\text {photon }}\right\rangle \approx 8.6 \mathrm{eV} /$ photon.

In the chamber, a $20 \mathrm{~mm}$ diameter metallic diaphragm was placed $5 \mathrm{~cm}$ away in front of the $\mathrm{MgF}_{2}$ window to stop the
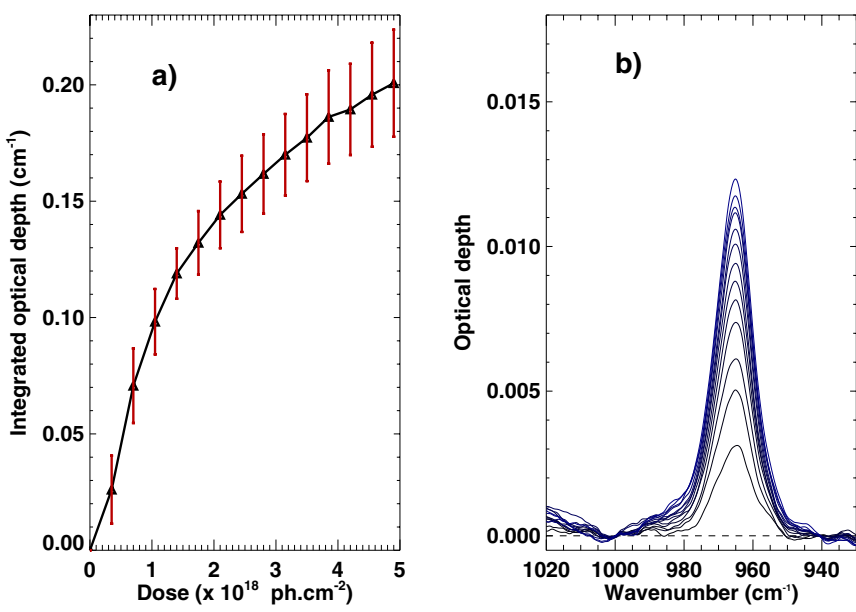

Fig. 3. a) Integrated absorption of the double bond in trans-vinylene group $(-\mathrm{HC}=\mathrm{CH}-)$ arising at $965 \mathrm{~cm}^{-1}$, as a function of dose, for irradiated polyethylene films placed in the substrate holder. The curve is the mean of two independent measurements with the corresponding error bars. b) Corresponding spectra showing the band increasing with fluence over the irradiation dose. These measurements ensure a regular actinometric calibration of the VUV lamp flux (see text for details).

photons that would otherwise irradiate other parts of the substrate holder or the chamber.

The VUV flux, defined as the number of photons per second per unit area, was obtained by using an actinometry measurement method. We used a polyethylene film, with a thickness of $15 \mu \mathrm{m}$ (Goodfellows), and replaced the HAC films on top of the substrate. This hydrocarbon polymer was chosen because it is well studied under irradiation with similar VUV sources (e.g., Truica-Marasescu \& Wertheimer 2005, and references therein). VUV irradiation gives rise to a new absorption band at $965 \mathrm{~cm}^{-1}$ attributed to the formation of double bonds from a trans-vinylene group $(-\mathrm{HC}=\mathrm{CH}-)$. The evolution of this band (Fig. 3b), well calibrated, gives access to the time-dependent dose at the exact same position where our interstellar analogs are subsequently processed. By scaling the time-dependent formation of transvinylene absorption to the calibrated measurement (Fig. 3a), we deduce a photon flux of $\Phi=2.7 \pm 0.6 \times 10^{14}$ photon $/\left(\mathrm{cm}^{2} \mathrm{~s}\right)$ in our experiment at the substrate position. This measurement was carried out regularly to monitor the potential degradation of the $\mathrm{MgF}_{2}$ lamp interface.

\subsection{QMS mass measurement}

The QMS was located at $\sim 10 \mathrm{~cm}$ from the film and aligned to make a $45^{\circ}$ angle with the film surface. A stable current of energetic electrons $(70 \mathrm{eV})$ was produced by a heated filament. These electrons ionize molecules and atoms with which they collide, and polarized plates accelerate the ions through a quadrupole mass filter. Ions with a certain mass-to-charge ratio propagate until they reach a deviation zone and are detected by a secondary-emission multiplier detector (SEM). In this particular configuration, VUV photons eventually reflected from the film holder, which behave as a source of noise, cannot reach the detector. To properly monitor the background signal when the UV lamp is on (i.e., the true zero of the signal, including possible contributions by photons that are absorbed or reflected outside the path to the sample), we placed a shutter in front of the film to allow periodic on-off measurements by stopping the incident photons on the film. This background was subtracted 
to the signal coming from the film. Two hundred masses were monitored simultaneously, with a mass resolution of $\frac{m}{\Delta m} \approx 50$. Without external calibration, the relative quantity of the corresponding species can, to first order, be related to the signal by taking into account the difference in ionization cross-sections for different species (at $70 \mathrm{eV}$ a factor of 3.5 is expected between $\mathrm{CH}_{4}$ and $\mathrm{H}_{2}$ ), and molecule fragments caused by electron impact-ionization (e.g., $\mathrm{CH}_{4}$ detected via $\mathrm{CH}_{4}^{+}, \mathrm{CH}_{3}^{+}, \mathrm{CH}_{2}^{+}$, $\mathrm{CH}^{+}, \mathrm{C}^{+}$and ${ }^{13} \mathrm{C}$ contribution), ionization cross-sections ratio are given at the NIST $^{1}$.

\subsection{Temperature-programed desorption for a-C:D and $\mathrm{a}-\mathrm{C}: \mathrm{H}$ films}

We began by cooling the shield down to $40 \mathrm{~K}$, while the temperature of the window was maintained at $200 \mathrm{~K}$ before the irradiation; this minimizes the condensation of residual gas onto the film. After that, the substrate window temperature was rapidly cooled down until it reached about $10.2 \mathrm{~K}$ and the film irradiation was started. After a period of irradiation at a fixed temperature $T$, a TPD (temperature-programed desorption) is performed with a heating ramp of 2.5 to $5 \mathrm{~K} / \mathrm{min}$, in the two cases of a-C:H and a-C:D analogs. During the $2.5 \mathrm{~K} / \mathrm{min}$ TPD, the VUV irradiation was kept on to avoid changing the experimental conditions. During the very first experiments, the ramps were stopped at $200 \mathrm{~K}$ and at up to $280 \mathrm{~K}$ in the following ones. Simultaneously, QMS measurements were carried out to follow the evolution of the species released from the film after irradiation.

\section{Results}

We present below the results obtained after the irradiation of several deuterated and HAC films prepared under the same conditions, and with variable thicknesses. They were irradiated at $10 \mathrm{~K}$ during several hours to simulate the conditions of an astrophysical dense cloud and produce the species in higher abundance than shorter irradiation times, so that we could perform quantitative measurements by comparing IR and mass-spectra measurements associated with a TPD program. Shorter irradiations were performed at higher temperatures $(50 \mathrm{~K}, 75 \mathrm{~K}, 100 \mathrm{~K}, 300 \mathrm{~K})$ to allow the diffusion out of the film to proceed efficiently, within the experiment duration, for the main produced species, which may otherwise seem to be trapped at the lowest temperatures because of the much shorter time scale than in space.

\subsection{IR measurement}

\subsubsection{Main features}

The absorption spectra in the infrared region between 4000 and $1000 \mathrm{~cm}^{-1}$ of the prepared a-C:H and a-C:D films are presented in Fig. 4. In a-C:H films we distinguish the bending modes of $\mathrm{C}-\mathrm{H}$ bonds between 1500 and $1300 \mathrm{~cm}^{-1}$ and stretching modes between 3100 and $2800 \mathrm{~cm}^{-1}$. There are four stretching modes; the asymmetric stretching modes in the methyl group $\mathrm{CH}_{3}$ and methylene group $\mathrm{CH}_{2}$ at about $2955 \mathrm{~cm}^{-1}$ and $2925 \mathrm{~cm}^{-1}$, and two symmetric modes for $\mathrm{CH}_{3}$ and $\mathrm{CH}_{2}$ groups at $2873 \mathrm{~cm}^{-1}$ and $2857 \mathrm{~cm}^{-1}$ (these two modes are blended, see Dartois et al. 2005).

For deuterium substitution the similar stretching modes are active, but they are shifted to lower frequencies, between 2015

\footnotetext{
1 http://www.nist.gov
}
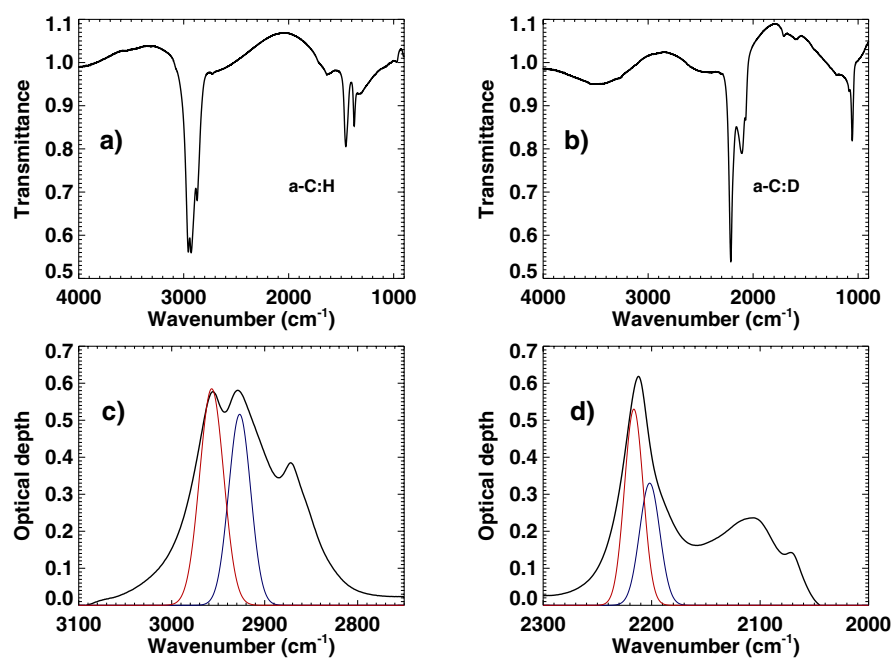

Fig. 4. a) Transmittance spectra of a-C:H films deposited on a $\mathrm{ZnSe}$ window at $10 \mathrm{~K}$. b) Transmittance spectra of a-C:D. c) Fits performed on the asymmetric $\mathrm{CH}_{3}$ and $\mathrm{CH}_{2}$ stretching modes. d) Fits performed on the asymmetric $\mathrm{CD}_{3}$ and $\mathrm{CD}_{2}$ stretching modes.

and $2285 \mathrm{~cm}^{-1}$, and the relative intensity between the modes is also changed. The asymmetric features from $\mathrm{CD}_{3}$ and $\mathrm{CD}_{2}$ are located at about 2220 and $2200 \mathrm{~cm}^{-1}$, while the symmetric modes are located at $2073 \mathrm{~cm}^{-1}$ and $2100 \mathrm{~cm}^{-1}$ (e.g., Tyrode \& Hedberg 2011).

The film thicknesses (Table 1) were estimated from the interference pattern. The fringe spacing $\Delta \sigma$ was measured and the sample thicknesses d were calculated using the formula

$d=1 /\left(2 n \Delta \sigma \cos \left(\alpha_{\mathrm{IR}}\right)\right)$,

with $n$ the refractive index of the sample, and $\alpha_{\mathrm{IR}}$ the angle of the IR beam incidence with the sample normal $\left(\alpha_{\mathrm{IR}}=45^{\circ}\right)$. Godard et al. (2010) measured the refractive index for several a-C:H samples. They found that $n$ varies between 1.25 and 1.85 . Our films have an estimated refractive index of $1.7 \pm 0.2$.

\subsection{2. a-C:H, a-C:D irradiation}

The total irradiation time for a-C:H and a-C:D films is typically five hours for short-duration and $18 \mathrm{~h}$ for long-irradiation times. The different irradiations by VUV photons at $10.2 \mathrm{~K}$ show the destruction of C-H and C-D bonds. To follow the evolution of the intensity of stretching modes and estimate the number of destroyed C-H bonds during the irradiation, an IR spectrum was recorded every $20 \mathrm{~min}$ with $15 \mathrm{~min}$ of coadded scans. The evolution of the optical depths integrated over the aliphatic C-H stretch band between 2760 and $3140 \mathrm{~cm}^{-1}$, as a function of the irradiation dose for different experiments, is represented in Fig. 6 (right axis).

The corresponding number of destroyed C-H bonds, $N_{\mathrm{C}-\mathrm{H}}$, is given by

$N_{\mathrm{C}-\mathrm{H}}=3 \times N_{\mathrm{CH}_{3}}+2 \times N_{\mathrm{CH}_{2}}=3 \times \frac{\int_{(\mathrm{as}) \mathrm{CH}_{3}} \Delta \tau \mathrm{d} \tau}{A_{\mathrm{CH}_{3}}^{\mathrm{as}}}+2 \times \frac{\int_{(\mathrm{as}) \mathrm{CH}_{2}} \Delta \tau \mathrm{d} \tau}{A_{\mathrm{CH}_{2}}^{\mathrm{as}}}$,

where $\Delta \tau$ is the variation in the optical depth after irradiation and $A_{\mathrm{CH}_{3}}^{\text {as }}, A_{\mathrm{CH}_{2}}^{\text {as }}$ are the integrated absorption coefficients of the antisymmetric stretching modes of $\mathrm{CH}_{3}$ and $\mathrm{CH}_{2}$ groups, with values equal to $1.25 \times 10^{-17}$ and $8.4 \times 10^{-18} \mathrm{~cm} /$ group (Dartois et al. 2004). 

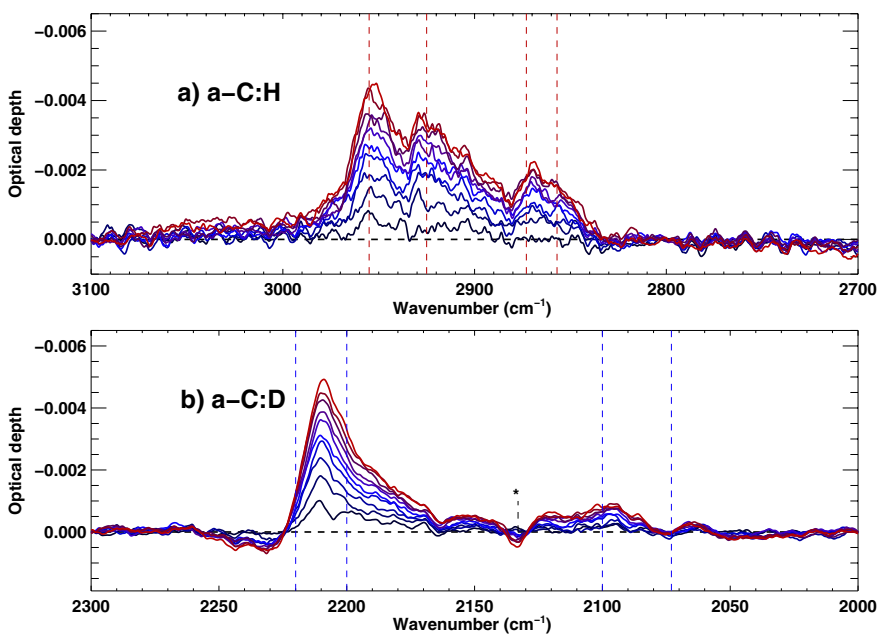

Fig. 5. Optical-depth decrease corresponding to different times of irradiation for a) a-C:H film in the $3100-2700 \mathrm{~cm}^{-1}$ range. b) a-C:D film in the $2300-2000 \mathrm{~cm}^{-1}$ range. Each curve corresponds to an additional irradiation time of $20 \mathrm{~min}$. The asterisk $*$ denotes solid CO contamination around $2133 \mathrm{~cm}^{-1}$

To evaluate the corresponding C-D bonds destruction, we evaluated to first order the corresponding band strengths for deuterated amorphous carbons by comparing the relative integrated absorptions in a-C:H and a-C:D films, normalized to the same film thickness. The fit was made only for the more intense asymmetric stretching modes, which are better separated than the symmetric ones for a-C:H. The asymmetric stretching features of a-C:H were fitted by three Gaussian profiles corresponding to the $\mathrm{CH}_{3}, \mathrm{CH}_{2}$, and $\mathrm{CH}_{2}$ Fermi resonance contributions, then grouped into two profiles related to their $\mathrm{CH}_{3}$ or $\mathrm{CH}_{2}$ character, as in Dartois et al. (2004). We proceeded in the same way for a-C:D films with the assigned band positions for the $\mathrm{CD}_{3}$ and $\mathrm{CD}_{2}$ asymmetric stretching modes, plus the contributions of $\mathrm{CD}_{2}$ Fermi resonances (e.g., Tyrode \& Hedberg 2011, band position assigned also by Raman spectroscopy). We show the corresponding profiles in Fig. 4. The a-C:D asymmetric stretching band strengths calculated in this work lead to $A\left(\mathrm{a}-\mathrm{CD}_{3}\right)=$ $9 \times 10^{-18} \mathrm{~cm} /$ group and $A\left(\mathrm{a}-\mathrm{CD}_{2}\right)=4.8-5.5 \times 10^{-18} \mathrm{~cm} /$ group . In a way similar to $N_{\mathrm{C}-\mathrm{H}}$, the number of destroyed C-D bonds C-D was estimated.

Figure 6 (left axis) shows the total number of destroyed C-H and C-D bonds as function of the number of photons. We can deduce that a $\mathrm{C}-\mathrm{H}$ bond is lost for about 70 photons (almost equal for C-D bond lost). This shows that this analog is particularly resistant to VUV irradiation. Part of this resistance with respect to other analogs is, in addition to the resilience of this material, related to its photoluminescence properties (Godard et al. 2010).

\subsection{Mass measurement}

In the following, we show that most of the $\mathrm{C}-\mathrm{H}$ bonds destruction leads to the production of $\mathrm{H}_{2}$ molecules, but also to small carbonaceous molecules. With our experimental conditions, $P \lesssim$ $2 \times 10^{-8}$ mbar, the quantity of measured $\mathrm{H}_{2}$ is dominated by the residual $\mathrm{H}_{2}$ that remains in the chamber after pumping. For this reason we also analyzed deuterated amorphous carbon films because the signal of $\mathrm{D}_{2}$ does not overlap with any other background signal. Photochemical properties of a-C:H and a-C:D are expected to behave similarly. Deuteration does not significantly change the electronic orbitals and electronic bonds in the material. During VUV photons irradiation, optically allowed

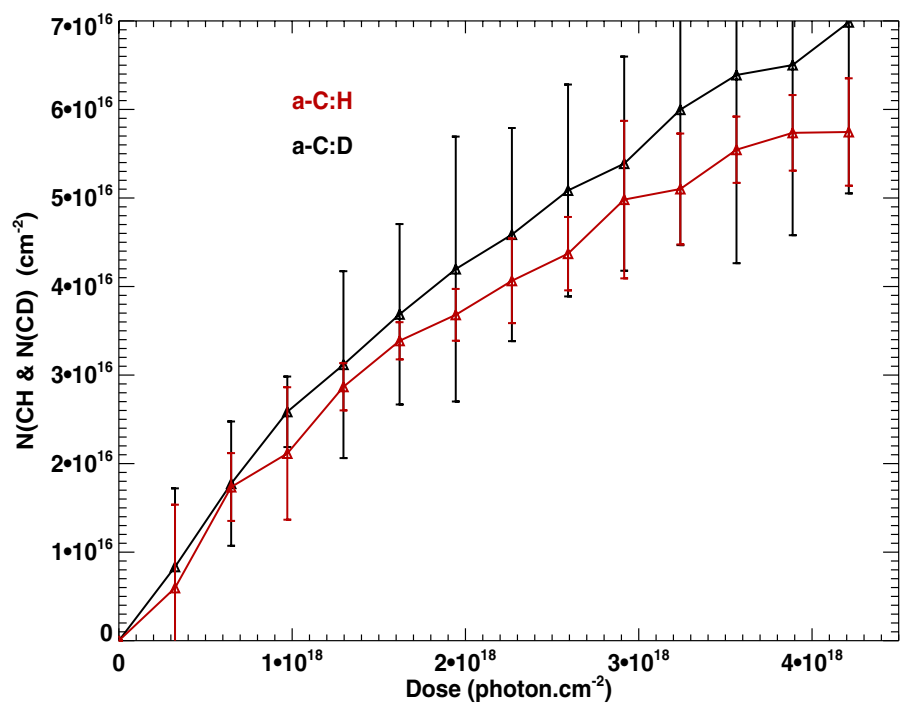

Fig. 6. Number of destroyed C-H bonds $N_{\mathrm{C}-\mathrm{H}}$ and C-D bonds $N_{\mathrm{C}-\mathrm{D}}$ as a function of the photon dose.

electronic singlet-singlet transitions are predominant at wavelengths of about $140 \mathrm{~nm}$ and above (Skurat 2003). Chemical transformations are mainly caused by reactions of these excited singlet states, which are very similar in both a-C:H and a-C:D materials. As was previously shown by IR spectroscopy (Fig. 6), the number of destroyed $\mathrm{C}-\mathrm{H}$ and $\mathrm{C}-\mathrm{D}$ bonds for the same irradiation dose are similar in both materials, within measurements uncertainties. Photoionization becomes competitive at higher energies, and the results are expected to be closer to radiolysis.

\subsection{1. $\mathrm{D}_{2}$ production}

The mass spectra of $\mathrm{D}_{2}(m / z=4 \mathrm{u})$, released from two a-C:D films and irradiated at $10 \mathrm{~K}$, during the TPD at a ramp rate of $2.5 \mathrm{~K} / \mathrm{min}$, are shown in panel a and $\mathrm{b}$ of Fig. 7. The mass $m / z=5 \mathrm{u}$ is presented as an adjacent background noise channel. In panel c, we present the TPD spectra of the same masses for an a-C:H film irradiated under the same conditions. $\mathrm{D}_{2}$ sublimation starts at a temperature of $\approx 17 \mathrm{~K}$ for the molecules produced closer to the surface and reaches maximum for the bulk at about $90-120 \mathrm{~K}$, well above the expected temperature of a surface $\mathrm{D}_{2}$ ice layer TPD behavior (e.g., Fillion et al. 2009). These $\mathrm{D}_{2}$ molecules were produced within the film during the VUV irradiation and diffuse from the bulk. For a-C:H, which served as a blank reference experiment, no signal was observed for these two masses.

The TPD desorption peak lies at a temperature above the expected physisorption barrier to move from site to site (inside, in the bulk, and at the surface), with a typical adsorption energy on surfaces for $\mathrm{D}_{2}$ of a few $10 \mathrm{meV}$. The TPD behavior is dominated by the permeation time needed to diffuse through the film, because the $D_{2}$ molecules are produced inside the bulk of the film for VUV irradiation. Additional experiments performed at 50, 75 and $100 \mathrm{~K}$ are shown in Fig. 8. The irradiation time windows are shown in the upper part of each panel. The intensities were normalized to the $\mathrm{D}_{2}$ signal when the film is irradiated at $300 \mathrm{~K}$ (last peak), a temperature at which the diffusion is almost instantaneous. When the irradiation stops, the signal decays as a function of the diffusion of $\mathrm{D}_{2}$ molecules out of the film. The diffusion can be estimated for the $100 \mathrm{~K}$ and $75 \mathrm{~K}$ experiments from the observed mean decay-time constant. Below about 50-60 K, 


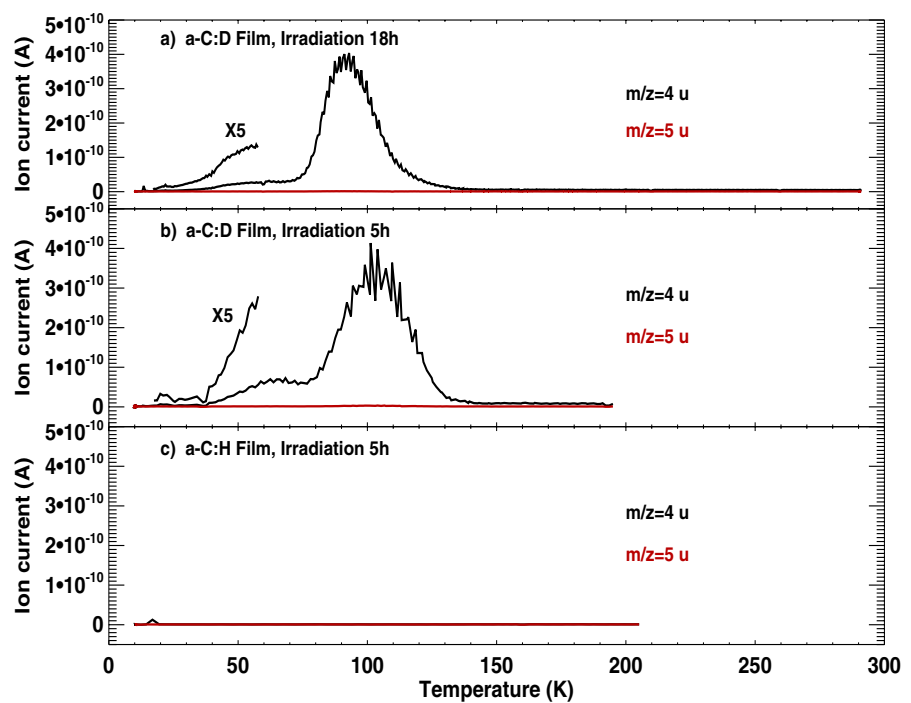

Fig. 7. a) TPD spectra of a-C:D film after $18 \mathrm{~h}$ of irradiation. $\mathrm{D}_{2}$ released from a-C:D film and the mass $m / z=5 \mathrm{u}$ as background mass channel. b) TPD spectra of a-C:D film after $5 \mathrm{~h}$ of irradiation. c) TPD spectra of a-C:H film after $5 \mathrm{~h}$ of irradiation. At mass $m / z=4 \mathrm{u}$ the signal is quite negligible and overlaps the background signal.

the diffusion decay- time constant becomes too low to be measured directly because of our pumping speed and QMS sensitivity limits. We define a typical decay-time constant $\tau$ as the time interval between the value of the $\mathrm{D}_{2}$ signal when the irradiation is stopped and the time when the signal has decayed to $10 \%$ of this value. At $100 \mathrm{~K}$, this corresponds to about $1500 \mathrm{~s}$, at $75 \mathrm{~K}$ it is about $4000 \mathrm{~s}$. The diffusion time constants generally follow an exponential behavior as a function of the inverse of the temperature. At $50 \mathrm{~K}$, the extrapolated decay-time constant would be of about $3 \times 10^{4} \mathrm{~s}$, at $25 \mathrm{~K}$ about $10^{7} \mathrm{~s}$ (three to four months), at $20 \mathrm{~K}$ about $1.8 \times 10^{8} \mathrm{~s}$ (about six years). These time constants are out of reach in the laboratory, but short in space.

\subsection{2. $\mathrm{CD}_{4}$ production}

The mass spectrum of $\mathrm{CD}_{4}(\mathrm{~m} / z=20 \mathrm{u})$ released from an irradiated a-C:D film during the TPD at a ramp rate of $2.5 \mathrm{~K} / \mathrm{min}$ is displayed in panel a of Fig. 9. The mass $m / z=21 \mathrm{u}$ is presented as the background mass channel, as well as the mass $m / z=18 \mathrm{u}$ (divided by 500) to check for possible contribution by water isotopologs. As for $\mathrm{D}_{2}$, a blank reference experiment with a-C:H was performed, monitoring the same channels, and is shown in panel b.

Two peaks are observed in the TPD of a-C:D film at $m / z=$ $20 \mathrm{u}$, a first peak located at about $153 \mathrm{~K}$, and a second one at $200 \mathrm{~K}$. In the a-C:H film case, only the first peak around $150 \mathrm{~K}$ is observed, slightly shifted compared with the corresponding a-C:D film one, and the second peak at $200 \mathrm{~K}$ is absent.

After several hours of irradiation, the peak at $m / z=18 \mathrm{u}$ arises around $150 \mathrm{~K}$ during the a-C:H TPD caused by the residual gas-phase water frozen onto cold surfaces during the experiment, under our high vacuum conditions. We clearly see the coincidence of the temporal evolution of this peak and the first peak that appears in the $m / z=20 \mathrm{u}$ of a-C:D TPD spectrum. Based on the a-C:H experiment, where the relative intensities of the peaks $((m / z=18 \mathrm{u}) /(m / z=20 \mathrm{u}) \approx 500)$ approximately correspond to the natural $\mathrm{O}^{18}$ to $\mathrm{O}^{16}$ isotope ratio, we attribute it to a contribution by $\mathrm{H}_{2}^{18} \mathrm{O}$. The second large peak that appears only in the a-C:D TPD spectrum at mass $m / z=20 \mathrm{u}$, with a
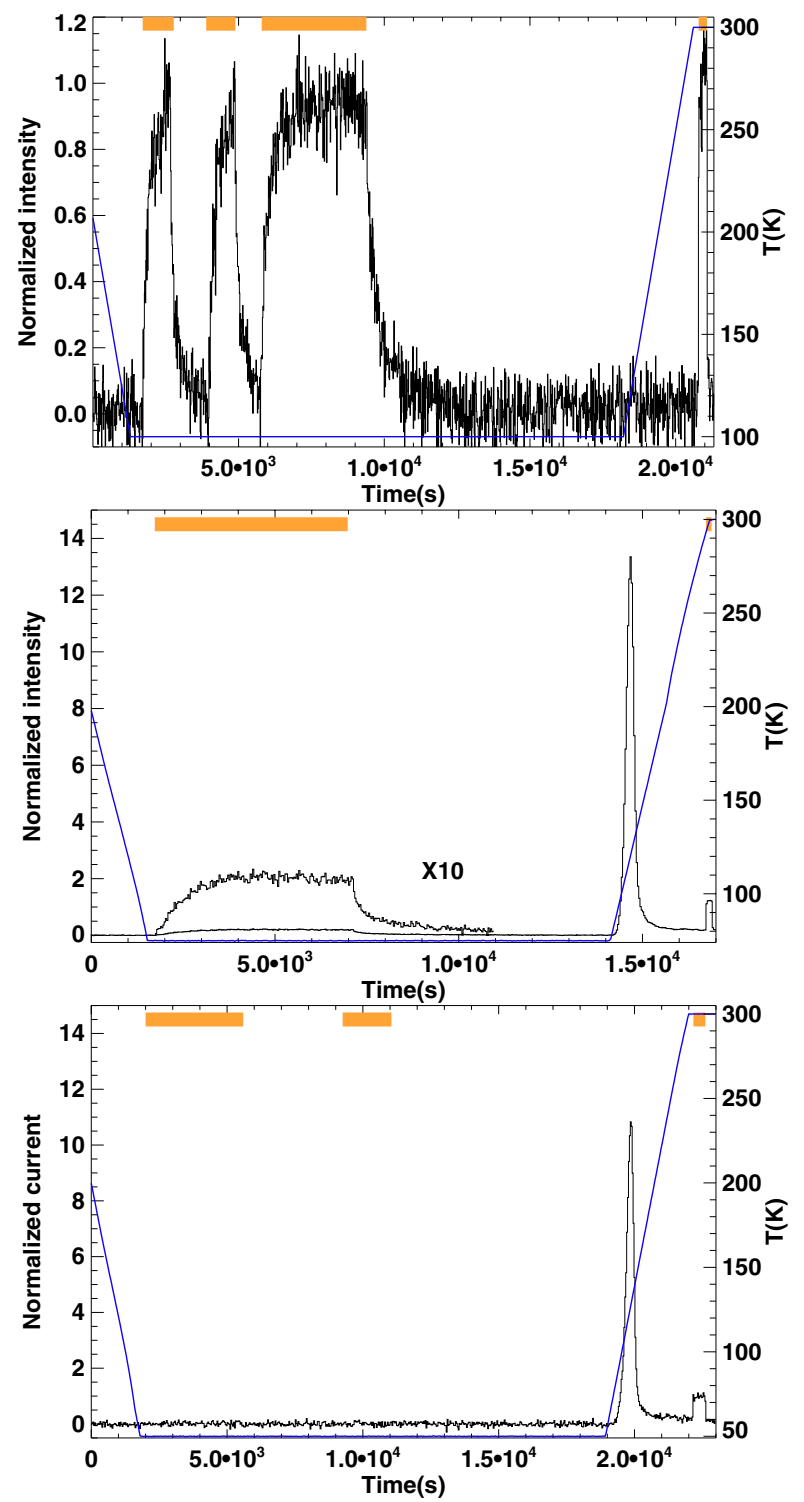

Fig. 8. Irradiation and TPD spectra of a-C:D film at $100 \mathrm{~K}$ (upper panel), $75 \mathrm{~K}$ (middle panel), and $50 \mathrm{~K}$ (lower panel). The irradiation sequences are shown with the upper bar and the blue curve gives the film temperature. See text for details.

maximum around $200 \mathrm{~K}$, is attributed to the $\mathrm{CD}_{4}$ produced by VUV photons within the film and diffusing from the bulk.

\subsubsection{Mass distribution}

To estimate the percentual abundance of each molecule, first we normalize each curve by the ionisation cross-section for each molecule, then we integrated each TPD curve signal. Systematics in the uncertainties are evaluated by first considering the respective contributions of the $\mathrm{D}_{2}$ and $\mathrm{CD}_{4}$ integrated curves, normalized to their sum, and then measuring the respective contributions of the $\mathrm{D}_{2}$ and $\mathrm{CD}_{4}$ integrated curve normalized to the sum of all integrated curves up to mass 60 . These two normalizations give us a minimum (including other masses) and a maximum (including only the desired signals) percentual abundance. We derived that $\mathrm{D}_{2}$ represents $95.8 \pm 3.4 \%$ of all the species released from the film, while $\mathrm{CD}_{4}$ represents about $3.1 \pm 2.1 \%$ The remaining signal is composed of other, mostly 


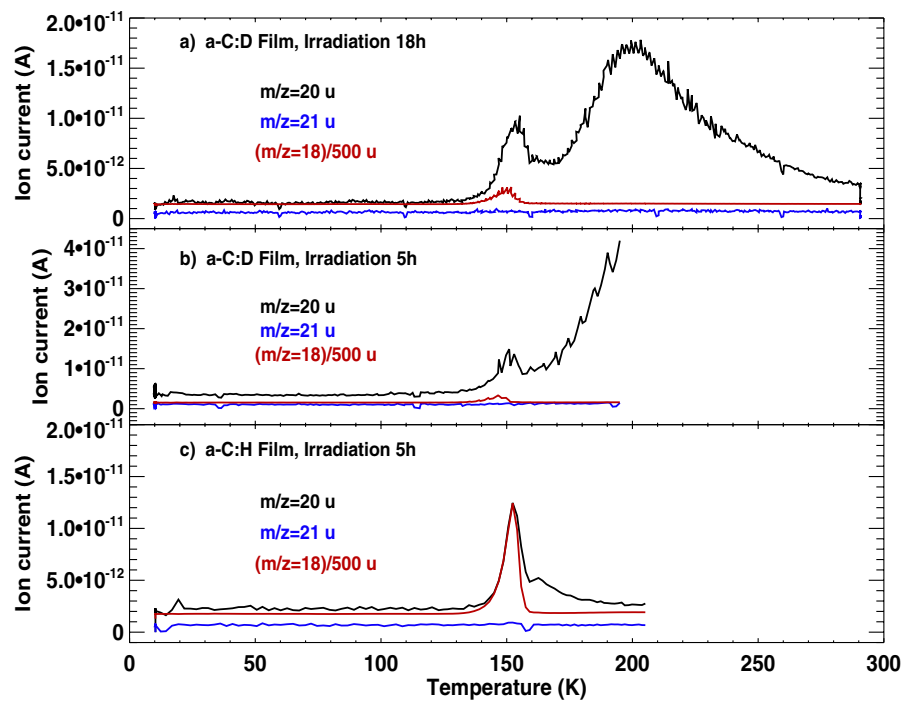

Fig. 9. a) TPD spectra of $C_{4}$ released from a-C:D film and the mass $m / z=21 \mathrm{u}$ as background mass channel. b) TPD spectra of a-C:D film after $5 \mathrm{~h}$ of irradiation. c) TPD spectra of a-C:H film, the two masses $\mathrm{m} / \mathrm{z}=20 \mathrm{u},(\mathrm{m} / \mathrm{z}=18) / 500$ and the mass $\mathrm{m} / \mathrm{z}=21 \mathrm{u}$ as background mass channel.

heavier, species under identification. This means that about $96 \%$ of the deuterium released by the destruction of C-D bonds is consumed by the formation of $\mathrm{D}_{2}$. We can deduce the total number of deuterated molecular hydrogen $N_{\mathrm{D}_{2}}$ from the number of destroyed C-D bonds $N_{\mathrm{C}-\mathrm{D}}^{\mathrm{d}}$,

$N_{\mathrm{D}_{2}}=\frac{1}{2} \times 0.958 \times N_{\mathrm{C}-\mathrm{D}}^{\mathrm{d}}=\gamma_{\mathrm{D} 2} \times N_{\mathrm{C}-\mathrm{D}}^{\mathrm{d}}$,

where $\gamma_{\mathrm{D} 2}$ is the $\mathrm{D}_{2}$ production-efficiency factor per photolyzed $\mathrm{CD}$ bond. For a-C:H we also estimated the quantity of $\mathrm{H}_{2}$ produced from the film in the same way.

\section{3. $\mathrm{H}_{2}$ quantum yield}

We define the differential quantum yield $Y_{\mathrm{H}_{2}}$ of molecular hydrogen production as the ratio of the number of produced molecules $N_{\mathrm{H}_{2}}$ formed from the sample per second per $\mathrm{cm}^{2}$ to the number of photons $\Phi$ absorbed by the sample per second per $\mathrm{cm}^{2}$,

$Y_{\mathrm{H}_{2}}=\frac{N_{\mathrm{H}_{2}}}{\Phi}$.

The integral quantum yield is defined as the ratio of the total number of product molecules formed in the sample over the irradiation time, thus corresponding to $Y_{\mathrm{H}_{2}}^{\text {int }}=\int Y_{\mathrm{H}_{2}}(t) \mathrm{d} t$.

Figure 10 presents the variation of differential quantum yields $Y_{\mathrm{H}_{2}}$ as a function of time. It decreases during irradiation. This decrease is explained not only by consumption (depletion) of $\mathrm{CH}_{3}$ and $\mathrm{CH}_{2}$ groups, but also by accumulation of conjugated photolysis products, which are able to "protect" the deeper layers by a mechanism of electronic energy transfer (e.g., Skurat 2003).

For thin films the photon flux is quite constant during propagation inside the film. We can accept in this case that the quantum yield is constant and close to the value when irradiation of the film is started, where $\mathrm{H}_{2}$ is produced mainly by the layers close to the surface; $Y_{\mathrm{H}_{2}} \simeq 0.025$. Absorbed photons do not all contribute to $\mathrm{C}-\mathrm{H}$ bond destruction. Part of the absorbed energy is dissipated by mechanisms like relaxation to electronic ground

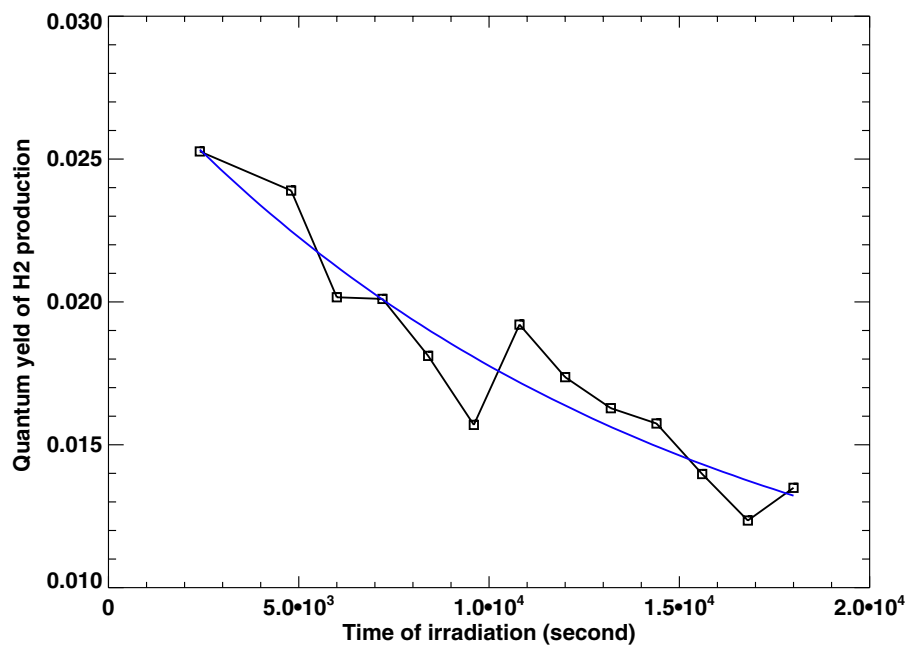

Fig. 10. Quantum yields of $\mathrm{H}_{2}$ production from a-C:H film, as a function of irradiation time.

state, photoluminescence (Godard et al. 2010) ${ }^{2}$, vibrational relaxation, or conical intersection of electronic states.

\section{Astrophysical implications}

Carbonaceous dust in the diffuse ISM is observed in emission via the PAH infrared emission bands and in absorption by a set of $\mathrm{CH}$ bond features at $3.4 \mu \mathrm{m}$ for the stretchings and 6.85 and $7.25 \mu \mathrm{m}$ for the bendings, associated with HACs. According to current models, PAHs require about $4-5 \%$ of the cosmic carbon abundance (e.g., Draine \& Li 2007) to exhibit the observed emission, whereas a-C:Hs (HACs) contain 5 to $30 \%$ of the cosmic carbon.

In our analysis of a-C:H submitted to VUV photolysis, we evaluated the effect of the decrease of the mean intensity of the photons in the film as a function of depth (e.g., Cottin et al. 2003, see their Fig. 4) using our films VUV absorption and the lamp spectral flux profile (Fig. 2), correcting for nonlinearities, and using different film thicknesses. The destruction cross-section is evaluated to be of the order of $\sigma_{\text {des }}=3 \pm 0.9 \times 10^{-19} \mathrm{~cm}^{2}$. This value is roughly 25 times higher than that obtained for a$\mathrm{C}: \mathrm{H}$ irradiation with much lower energy photons $\left(\approx \sigma_{\text {des }}=1.2 \times\right.$ $10^{-20} \mathrm{~cm}^{2}$ ) estimated by Mennella (2001) from the experiments reported by Ida et al. (1984).

It can be compared with the VUV irradiation of various hydrocarbon species (Muñoz Caro et al. 2001) and hydrogenated carbon grains produced by condensation of carbon vapour obtained by striking an arc discharge between two carbon rods in a hydrogen atmosphere (Mennella et al. 1999, 2001). For the latter, a UV photodestruction cross-section of $\sigma_{\text {des }}=1$ to $5 \times 10^{-19} \mathrm{~cm}^{2}$ per photon was estimated from the IR, assuming a simple two layer hydrocarbon model, decoupling an unprocessed zone at high depth from a UV irradiated one closer to the surface. We thus find similar destruction cross-sections for the aliphatic $\mathrm{C}-\mathrm{H}$ bonds in interstellar analogs made under different conditions. In this work, we show by using a mass-spectrometric detection method that the main released product of a-C:H photolysis is molecular hydrogen, with a cross-section of $\mathrm{H}_{2}$ production of $\sigma_{\mathrm{H}_{2} \text { prod }} \approx 1.4 \times 10^{-19} \mathrm{~cm}^{2} /$ photon.

2 High values for the photoluminescence quantum yields $\eta_{\mathrm{PL}}$ have been measured in the near UV and visible region for a-C: $\mathrm{H}$ films $\left(\eta_{\mathrm{PL}}=\right.$ $7 \%$ at $250 \mathrm{~nm})$. 
These high destruction cross-sections entail a short lifetime for hydrogenated carbonaceous dust grains, which are not expiated to survive the diffuse-medium UV field, if one only takes into account the destruction. The presence of these grains in the diffuse medium therefore implies that there must be rehydrogenation or reformation pathways for the $\mathrm{CH}$ bonds in space, otherwise there would be no interstellar detection of aCH. Mennella (1999) found that the C-H mode can be reactivated (i.e., the grain rehydrogenated) by exposure to a beam of $\mathrm{H}$ atoms. He showed by a simple modeling based on these experiments that the observed a-C:H is expected be hydrogenated constantly in the diffuse medium, otherwise, as stated above, the photolysis time-scale of HACs would rapidly dehydrogenate them. Possible reactivation processes for $\mathrm{CH}$ bonds were also discussed in Muñoz Caro et al. (2001) and Mennella et al. (2001). Mennella (2006) performed experiments to determine an activation energy of about $70 \mathrm{~K}$.

Early studies (Gould \& Salpeter 1963; Hollenbach \& Salpeter 1971a; Hollenbach et al. 1971b; Jura 1975) provided the first estimates of $\mathrm{H}_{2}$ formation rates in the diffuse ISM, concluding that grain surface chemistry is an unavoidable route for efficient hydrogen formation. These early hydrogen-formation rate numbers are within small factors still the ones used today. Hydrogen has the highest cosmic abundance. Several mechanisms are responsible for the formation of molecular hydrogen in the ISM; their importance varies depending on the environmental conditions.

Several laboratories have worked on $\mathrm{H}$ atom irradiation and adsorption on diverse surfaces such as graphite and other carbonaceous material, silicates, and ice mantles (mainly water ice), followed by chemistry leading to $\mathrm{H}_{2}$ production (e.g. Gavilan et al. 2012; Vidali et al. 2009; Mennella 2008; Amiaud et al. 2007; Perets 2007; Creighan et al. 2006, and references therein). Many of these studies focused principally on physisorbed atoms and are in general poorly efficient in producing large $\mathrm{H}_{2}$ abundances at temperatures above about $30 \mathrm{~K}$. The observation of $\mathrm{H}_{2}$ formation in astronomical environments with gas at high kinetic temperatures has led to the proposition of a mechanism that necessarily involves chemisorbed $\mathrm{H}$ atoms (Habart et al. 2005; Cazaux et al. 2011; Le Bourlot et al. 2012) to overcome the barrier of higher temperature formation, in particular in PDR environments. The rather constant hydrogen rate coefficient $\left(R_{\mathrm{c}}\right)$ deduced in astronomical source lines of sight with degrees of excitation varying over a wide range is a severe constraint for thermally activated processes.

Alternative studies have searched for the influence of cosmic rays on the $\mathrm{H}_{2}$ formation in denser regions (e.g., Pirronello et al. 1997), showing that in shielded regions this is a valid competing way for producing $\mathrm{H}_{2}$. Mennella et al. (2003) and Godard et al. (2011) have also followed the destruction cross-section by cosmic rays. These processes also produce $\mathrm{H}_{2}$, but are probably less efficient than the UV in the diffuse medium and the PDR interfaces.

Many of the studies cited above have been performed ignoring the energetic impact of ultraviolet photons, or considering their energy input almost solely for the destruction rate (photodissociation) of $\mathrm{H}_{2}$ in the gas phase, to balance the formation rate equation and deduce it from the observations.

\subsection{Comparison with the surface formation rate coefficient}

The observation of the so-called $3.4 \mu \mathrm{m}$ band in the diffuse ISM also results from the balance between destruction, with $\mathrm{H}_{2}$ as a byproduct, and hydrogenation. The mean penetration depth for the VUV photons produced by a hydrogen discharge lamp $(120-160 \mathrm{~nm})$ is approximately $80 \mathrm{~nm}$, thus clearly affecting the bulk of the grains. It allows the formation of $\mathrm{H}_{2}$ mediated via the bulk of the grains as opposed to pure surface schemes.

From our measurements, a photolytic destruction rate of $\mathrm{CH}$ bonds in grains can be evaluated,

$R_{\mathrm{des}}^{\mathrm{FUV}}\left[\mathrm{cm}^{-3} \mathrm{~s}^{-1}\right]=\int_{\mathrm{FUV}} \sigma_{\mathrm{CH}}^{\mathrm{des}}(\lambda) \frac{n(\mathrm{CH})}{n_{\mathrm{tot}}} n_{\mathrm{tot}} \phi(\lambda) \mathrm{d} \lambda$,

where $\sigma_{\mathrm{CH}}^{\mathrm{des}}(\lambda)$ is in $\mathrm{cm}^{2}$ photon ${ }^{-1} \mathrm{CH}^{-1}$ and $\phi(\lambda)$ in photon $\mathrm{cm}^{-2} \mathrm{~s}^{-1} \AA^{-1}$,

Because most of the VUV photon penetration length is lower than or equal to the grain size, this can be approximated by (see also Mennella 2002)

$R_{\mathrm{des}}^{\mathrm{FUV}} \approx \sigma_{\mathrm{CH}}^{\mathrm{des}}(\mathrm{FUV}) \frac{n(\mathrm{CH})}{n_{\mathrm{tot}}} n_{\mathrm{tot}} \phi(\mathrm{FUV})$,

where $\sigma_{\mathrm{CH} \text { des }}(\mathrm{FUV})$ and $\phi(\mathrm{FUV})$ are the integrated destruction cross-section in $\mathrm{cm}^{2}$ photon ${ }^{-1} \mathrm{CH}^{-1}$ and $\mathrm{UV}$ flux in photon $\mathrm{cm}^{-2} \mathrm{~s}^{-1}$,

The corresponding photolytic $\mathrm{H}_{2}$ formation rate reads

$$
R_{\mathrm{f}}^{\mathrm{FUV}}\left[\mathrm{cm}^{-3} \mathrm{~s}^{-1}\right] \approx \gamma_{\mathrm{H} 2} \sigma_{\mathrm{CH}}^{\mathrm{des}}(\mathrm{FUV}) \frac{n(\mathrm{CH})}{n_{\mathrm{tot}}} n_{\mathrm{tot}} \chi \phi^{\mathrm{ISRF}}(\mathrm{FUV}) \mathrm{e}^{-\tau_{\mathrm{d}}}
$$

where $\gamma_{\mathrm{H} 2}$ is the $\mathrm{H}_{2}$ production efficiency factor per photolyzed $\mathrm{CH}$ bond, $\phi^{\mathrm{ISRF}}$ (FUV) is the standard interstellar radiation field FUV flux photon $\mathrm{cm}^{-2} \mathrm{~s}^{-1}, \chi$ is a scaling factor (Draine1978), $\frac{[n(\mathrm{CH})]}{\left[n_{\mathrm{tot}}\right]}=f_{\mathrm{aCH}}^{\mathrm{C}} \times \frac{[\mathrm{C}]}{[\mathrm{H}]}, f_{\mathrm{aCH}}^{\mathrm{C}}$ the fraction of interstellar carbon abundance locked into a-C:H, and $n_{\text {tot }}=n(\mathrm{H})+2 n_{\mathrm{H}_{2}}$ is the total number density of hydrogen atoms, and $\mathrm{e}^{-\tau_{\mathrm{d}}}$ is the dust extinction.

The seminal equation for the $\mathrm{H}_{2}$ formation rate derives generally from a consensus that the molecule forms from the recombination on the surface of dust grains from gas-phase incoming $\mathrm{H}$ atoms (e.g., Hollenbach et al. 1971b). Therefore it is by definition proportional to the atomic $\mathrm{H}$ number density and the total number density, with an assumed gas-to-grain ratio. The formation rate is the product of a rate coefficient times these number densities. The $R_{\mathrm{f}}^{\mathrm{FUV}}$ formation rate can be rewritten to extract a rate coefficient for this VUV-induced mechanism that can be set in correspondence to the one used in the rate equation of hydrogen recombination on a surface,

$R_{\mathrm{f}}^{\mathrm{FUV}}\left[\mathrm{cm}^{-3} \mathrm{~s}^{-1}\right]=R_{\mathrm{c}}\left[\mathrm{cm}^{3} \mathrm{~s}^{-1}\right] n(\mathrm{H}) n_{\mathrm{tot}}$.

This formulation allows performing more direct comparisons of rate coefficients of $\mathrm{H}_{2}$ formation generated in the bulk by VUV with those obtained from hydrogen recombination on the surface of dust grains due to $\mathrm{H}$ atoms coming from the gas-phase. Therefore, we can assess the importance of the bulk photolysis that is the aim of this investigation. Proceeding from the previous equations, then

$R_{\mathrm{c}}=\gamma_{\mathrm{H} 2} \sigma_{\mathrm{CH}}^{\mathrm{des}}(\mathrm{FUV}) f_{\mathrm{aCH}}^{\mathrm{C}} \times \frac{[\mathrm{C}]}{[\mathrm{H}]} \chi \phi^{\mathrm{ISRF}}(\mathrm{FUV}) \mathrm{e}^{-\tau_{\mathrm{d}}} / n(\mathrm{H})$
$\gamma_{\mathrm{H} 2}=\frac{1}{2} \times 0.958, \sigma_{\mathrm{CH}}^{\mathrm{des}}(\mathrm{FUV})=3 \times 10^{-19} \mathrm{~cm}^{2}, \frac{[\mathrm{C}]}{[\mathrm{H}]}=3.55 \times 10^{-4}$,

The standard FUV $(6<\mathrm{h} v<13.6 \mathrm{eV})$ interstellar radiation field from Draine (1978) corresponds to $\phi^{\mathrm{ISRF}}(\mathrm{FUV})=$ $1.94 \times 10^{8}$ photon $\mathrm{cm}^{-2} \mathrm{~s}^{-1}\left(2.68 \times 10^{-3} \mathrm{erg} \mathrm{s}^{-1} \mathrm{~cm}^{-2}\right.$, that is about 1.7 times $\mathrm{G}_{0}$, the standard ISRF from Habing 1968),

$R_{\mathrm{c}}\left[\mathrm{cm}^{3} \mathrm{~s}^{-1}\right] \approx 9.9 \times 10^{-15} f_{\mathrm{aCH}}^{\mathrm{C}} \chi \mathrm{e}^{-\tau_{\mathrm{d}}} / n(\mathrm{H})$, 
If only $10 \%$ of the carbon abundance is locked into HACs, $f_{\mathrm{aCH}}^{\mathrm{C}}=$ 0.1 , and

$$
R_{\mathrm{c}}\left[\mathrm{cm}^{3} \mathrm{~s}^{-1}\right] \approx 9.9 \times 10^{-16} \times \mathrm{e}^{-\tau_{\mathrm{d}}} / n(\mathrm{H}) .
$$

This rate must be compared with the adopted rate coefficient of $R_{\mathrm{c}} \approx 3 \times 10^{-17} \mathrm{~cm}^{3} \mathrm{~s}^{-1}$ from observations in the diffuse medium (e.g., Jura 1974; Gry et al. 2002) with $\chi / n(\mathrm{H}) \approx 1 / 30$, and to PDRs $^{3}$, where $R_{\mathrm{c}}$ can rise to $\approx 1.5 \times 10^{-16} \mathrm{~cm}^{3} \mathrm{~s}^{-1}$ (e.g., Habart et al. 2004). The $\chi / n(\mathrm{H})$ in these regions reaches values of up to 0.25 (e.g., Orion Bar).

The photolytic bulk production of $\mathrm{H}_{2}$ with carbonaceous HAC dust grains is thus able to sustain a very large portion of the contribution to the $\mathrm{H}_{2}$ formation, and probably all the necessary rate formation in some PDRs.

\subsection{Implications for molecular abundances}

The equations were rewritten above to compare the derived FUV formation rate with previous works. There is a significant difference between models assuming a surface reaction scheme (either physisorbed or chemisorbed) with models of FUV photolysis. In the seminal equation equilibrating the formation and destruction rates, when $\mathrm{H}_{2}$ is produced by UV photolysis in the bulk of the grains, the dust grain UV flux attenuation appears on both sides of the equation, and thus, the dust extinction equally affects the formation and destruction rate:

$$
\begin{aligned}
R_{\mathrm{f}}^{\mathrm{FUV}}\left[\mathrm{cm}^{-3} \mathrm{~s}^{-1}\right] \approx \gamma_{\mathrm{H} 2} & \sigma_{\mathrm{CH}}^{\mathrm{des}}(\mathrm{FUV}) \frac{n(\mathrm{CH})}{n_{\text {tot }}} n_{\mathrm{tot}} \chi \phi^{\mathrm{ISRF}}(\mathrm{FUV}) \mathrm{e}^{-\tau_{\mathrm{d}}} \\
& =\operatorname{Rd}_{\mathrm{H}_{2}}(0) \chi \mathrm{e}^{-\tau_{\mathrm{d}}} f_{\mathrm{s}}\left(N\left(\mathrm{H}_{2}\right)\right) n_{\mathrm{H}_{2}},
\end{aligned}
$$

where $\mathrm{Rd}_{\mathrm{H}_{2}}(0)$ is the unshielded photodissociation rate per $\mathrm{H}_{2}$ for $\chi=1$, given by $\sim 5 \times 10^{-11} \mathrm{~s}^{-1}$, see, for example Eq. (1) in Habart et al. (2004) and $f_{\mathrm{s}}\left(N\left(\mathrm{H}_{2}\right)\right)$ the $\mathrm{H}_{2}$ self-shielding factor at a given $N\left(\mathrm{H}_{2}\right)\left[\mathrm{cm}^{2}\right]$ column density from the FUV illuminating source. The molecular $\mathrm{H}_{2}$ abundance is therefore given by

$$
\frac{n_{\mathrm{H}_{2}}}{n_{\mathrm{tot}}}=\frac{\gamma_{\mathrm{H} 2} \sigma_{\mathrm{CH}}^{\mathrm{des}}(\mathrm{FUV}) \frac{n(\mathrm{CH})}{n_{\mathrm{tot}}} \phi^{\mathrm{ISRF}}(\mathrm{FUV})}{\operatorname{Rd}_{\mathrm{H}_{2}}(0) f_{\mathrm{s}}\left(\mathrm{N}_{(}\left(\mathrm{H}_{2}\right)\right)}
$$

with the above numbers. This implies for the very diffuse unshielded ISM that the smallest molecular fraction is

$\left(\frac{n_{\mathrm{H}_{2}}}{n_{\mathrm{tot}}}\right)_{\mathrm{ISM}} \sim 10^{-5} f_{\mathrm{aCH}}^{\mathrm{C}}$,

with $f_{\mathrm{aCH}}^{\mathrm{C}}$ the fraction of interstellar carbon abundance locked into a-C:H. This can be compared with the observations for atomic diffuse clouds, such as high-latitude lines of sights (e.g., Wakker 2006; Richter et al. 2003).

The FUV photolysis of bulk carbonaceous dust grains is not only able to provide the ISM with $\mathrm{H}_{2}$ molecules, but, as shown in our experiments, also delivers small hydrocarbons such as methane to the gas phase. This is accompanied by a network cross-linking and structural modification (e.g., Gadallah et al. 2011). This gas-phase released $\mathrm{CH}_{4}$ will be efficiently photodissociated (photodissociation rate $\approx 1.2 \times 10^{-9} \mathrm{~s}^{-1}$, van Dishoeck et al. 2006). The small hydrocarbons produced from this methane release will contribute to the abundance observed in the diffuse ISM (e.g., Liszt et al. 2012), a consequence

\footnotetext{
3 Dividing by two $R_{\mathrm{c}}^{\mathrm{FUV}}\left(\mathrm{H}_{2}\right)\left[\mathrm{cm}^{3} \mathrm{~s}^{-1}\right] \approx 4.86 \times 10^{-16} \chi / n(\mathrm{H})$, to take into account that only half the space receive the photon flux for a semiinfinite cloud and an isotropic impinging radiation field, as explained in Le petit et al. (2006).
}

of the erosion of a-C:H grains under the effect of the FUV photolysis.

The steady-state molecular $\mathrm{CH}_{4}$ abundance can be estimated, as for $\mathrm{H}_{2}$, from

$$
\frac{n_{\mathrm{CH}_{4}}}{n_{\mathrm{tot}}}=\frac{\gamma_{\mathrm{CH}_{4}} \sigma_{\mathrm{CH}}^{\mathrm{des}}(\mathrm{FUV}) \frac{n(\mathrm{CH})}{n_{\text {tot }}} \phi^{\mathrm{ISRF}}(\mathrm{FUV})}{\operatorname{Rd}_{\mathrm{CH}_{4}}(0) \mathrm{f}_{\mathrm{s}}\left(\mathrm{N}\left(\mathrm{CH}_{4}\right)\right)} \text {, }
$$

where $\mathrm{Rd}_{\mathrm{CH}_{4}}(0)$ is the unshielded photodissociation rate per $\mathrm{CH}_{4}$ for $\chi=1$. This implies for the very diffuse unshielded ISM that the smallest molecular fraction reaches, with $\gamma_{\mathrm{CH}_{4}}=\frac{1}{4} \times 0.031=$ 0.00775 ,

$$
\left(\frac{n_{\mathrm{CH}_{4}}}{n_{\mathrm{tot}}}\right)_{\mathrm{ISM}} \sim 1.3 \times 10^{-7} f_{\mathrm{aCH}}^{\mathrm{C}},
$$

The photoproduced $\mathrm{CH}_{4}$ is released from the a-C:H network at a higher temperature than $\mathrm{H}_{2}$ in the TPD experiments. Given this slower diffusion rate, $\mathrm{CH}_{4}$ may be photodissociated while diffusing in the grain over astrophysical time scales. This matter deserves additional experimental investigation.

\section{Conclusion}

We have experimentally investigated the VUV photolysis of HACs, analogs of the interstellar a-C:H. We showed by combining IR to mass spectrometry that the FUV irradiation leads to the efficient production of $\mathrm{H}_{2}$ molecules, but also to small hydrocarbons such as $\mathrm{CH}_{4}$, released from the a-C:H film.

The species are produced by VUV photolysis not only at the surface, but principally within the bulk of the a-C:H carbonaceous network and diffuse out at higher temperatures than the purely physisorbed species made by the recombination of $\mathrm{H}$ atoms on surfaces. This provides efficient formation in environments where the residence time scale for $\mathrm{H}$ atoms prevents an efficient formation on the surface. This mechanism provides high $\mathrm{H}_{2}$ formation rates at low to high grain temperatures.

In an interstellar context where both $\mathrm{H}$ atoms and FUV photons are present, this reaction can be viewed as a catalytic production with a repeated atomic $\mathrm{H}$-addition and VUV $\mathrm{H}$-abstraction on the same grains. This photolytic process will progressively erode the carbon network, however, and the carbon loss is not fully reversible because hydrocarbons molecules are produced and ejected in the photochemical process.

At the interface of PDRs, an interesting consequence on the observed rate coefficient is the grain hydrogenation balance driven by how fast the rehydrogenation of the grains proceeds and/or how fast the process is advective, that is processes newly exposed grains, because real PDRs are not in a steady state, as assumed in most models.

The a-C:H material feeds the ISM with small hydrocarbons, which contribute to the formation of small carbonaceous radicals after being dissociated by the UV photons in the considered environment.

We experimentally investigated only the first small hydrocarbon, $\mathrm{CH}_{4}$, produced during the VUV photolysis of a-C:H. UHV experiments are currently conducted to extend the analyses to a quantitative information on heavier species that are released into the gas phase and feed the backend of the ladder for the formation of more complex hydrocarbons or carbon-based radicals that are detected at radio wavelengths.

Acknowledgements. This work was supported by the ANR COSMISME project, grant ANR-2010-BLAN-0502 of the French Agence Nationale de la Recherche. Part of the equipment used in this work has been financed by the ANR and 
French INSU-CNRS program "Physique et Chimie du Milieu Interstellaire" (PCMI). G.A.C.D. and G.M.M.C. were financed by Spanish MINECO projects AYA2011-29375 and CONSOLIDER grant CSD 2009-00038. We thank the anonymous referee for the comments that helped to improve the manuscript.

\section{References}

Acke, B., \& van den Ancker, M. E. 2006, A\&A, 457, 171

Acke, B., Bouwman, J., Juhász, A., et al. 2010, ApJ, 718, 558

Allen, D. A., \& Wickramasenghe, D. T. 1981, Nature, 294, 239

Allamandola, L. J., Tielens, A. G. G. M., \& Barker, J. R. 1985, ApJ, 290, L25

Amiaud, L., Dulieu, F., Fillion, J.-H., Momeni, A., \& Lemaire, J. L. 2007, J. Chem. Phys., 127, 144709

Boersma, C., Bouwman, J., Lahuis, F., et al. 2008, A\&A, 484, 241

Butchart, I., McFadzean, A. D., Whittet, D. C. B., Geballe, T. R., \& Greenberg,

J. M. 1986, A\&A, 154, L5

Carpentier, Y., Féraud, G., Dartois, E., et al. 2012, A\&A, 548, A40

Cazaux, S., Morisset, S., Spaans, M., \& Allouche, A. 2011, A\&A, 535, A27

Chang, H. C., Lin, J. C., Wu, J. Y., \& Chen K. H. 1995, J. Phys. Chem., 99, 11081

Chiar, J. E., Adamson, A. J., Pendleton, Y. J., et al. 2002, ApJ, 570, 198

Cottin, H., Moore, M. H., \& Bénilan, Y. 2003, ApJ, 590, 874

Creighan, S. C., Perry, J. S. A., \& Price, S. D. 2006, J. Chem. Phys., 124, 114701

Cruz-Diaz, G. A., Muñoz Caro, G. M., \& Chen, Y. J. 2013, A\&A, accepted

Dartois, E., Marco, O., Muñoz-Caro, G. M., et al. 2004, A\&A, 423, 549

Dartois, E., Muñoz Caro, G. M., Deboffle, D., Montagnac, G., \& D'Hendecourt,

L. 2005, A\&A, 432, 895

Draine, B. T. 1978, ApJ, 36, 595

Draine, B. T., \& Li, A. 2007, ApJ, 657, 810

Duley, W. W. 1994, ApJ, 430, L133

Duley, W. W., \& Williams, D. A. 1983, MNRAS, 205, 67

Duley, W. W., Scott, A. D., Seahra, S., \& Dadswell, G. 1998, ApJ, 503, L183

Ehrenfreund, P., Robert, F., D'Hendecourt, L., \& Behar, F. 1991, A\&A, 252, 712

Fillion, J.-H., Amiaud, L., Congiu, E., et al. 2009, Phys. Chem. Chem. Phys., 11,4396

Furton, D. G., Laiho, J. W., \& Witt, A. N. 1999, ApJ, 526, 752

Gadallah, K. A. K., Mutschke, H., Jaumlger, C. 2011, ApJ, 528, A56

Gadallah, K. A. K., Mutschke, H., \& Jager, C. 2013, A\&A, 554, A12

Gavilan, L., Lemaire, J. L., \& Vidali, G. 2012, MNRAS, 424, 2961

Geballe, T. R., Chiar, J., Pendleton, Y. J., \& Tielens, A. G. G. M. 1998, Ap\&SS, 255,457

Godard, M., \& Dartois, E. 2010, A\&A, 519, A39

Godard, M., Féraud, G., Chabot, M., et al. 2011, A\&A, 529, A146

Godard, M., Geballe, T. R., Dartois, E., \& Muñoz Caro, G. M. 2012, A\&A, 537, A27

Goto, M., Henning, T., Kouchi, A., et al. 2009, ApJ, 693, 610

Gould, R. J., \& Salpeter, E. E. 1963, ApJ, 138, 393

Gry, C., Boulanger, F., Nehmé, C., et al. 2002, A\&A, 391, 675

Guillois, O., Ledoux, G., \& Reynaud, C. 1999, ApJ, 521, L133

Habart, E., Walmsley, M., Verstraete, L., et al. 2005, Space Sci. Rev., 119, 71

Habart, E., Boulanger, F., Verstraete, L., Walmsley, C. M., \& Pineau des Forêts, G. 2004a, A\&A, 414, 531

Habart, E., Testi, L., Natta, A., \& Carbillet, M. 2004b, ApJ, 614, L129

Habing, H. J. 1968, Bull. Astron. Inst. Netherlands, 19, 421
Hollenbach, D., \& Salpeter, E. E. 1971, ApJ, 163, 155

Hollenbach, D. J., Werner, M. W., \& Salpeter, E. E. 1971, ApJ, 163, 165 Imanishi, M. 2006, AJ, 131, 2406

Imanishi, M., Nakagawa, T., Ohyama, Y., et al. 2008, PASJ, 60, 489

Iida, S., Ohtaki, T., \& Seki, T. 1984, in Optical Effects in Amorphous Semiconductors, eds. P. C. Taylor, \& S. G. Bishop (New York: AIP), AIP Conf. Proc. 120, 258

Jones, T. J., Hyland, A. R., \& Allen, D. A. 1983, MNRAS, 205, 187

Jones, A. P., Fanciullo, L., Köhler, M., et al. 2013, A\&A, 558, A62

Jura, M. 1974, ApJ, 191, 375

Jura, M. 1975, ApJ, 197, 575

Keller, L. D., Sloan, G. C., Forrest, W. J., et al. 2008, ApJ, 684, 411

Le Bourlot, J., Le Petit, F., Pinto, C., Roueff, E., \& Roy, F. 2012, A\&A, 541, A76

Lee, W., \& Wdowiak, T. J. 1993, ApJ, 417, L49

Leger, A., \& Puget, J. L. 1984, A\&A, 137, L5

Liszt, H., Sonnentrucker, P., Cordiner, M., \& Gerin, M. 2012, ApJ, 753, L28

Le Petit, F., Nehmé, C., Le Bourlot, J., \& Roueff, E. 2006, ApJS, 164, 506

Mason, R. E., Wright, G., Pendleton, Y., \& Adamson, A. 2004, ApJ, 613, 770

McFadzean, A. D., Whittet, D. C. B., Bode, M. F., Adamson, A. J., \& Longmore,

A. J. 1989, MNRAS, 241, 873

Mennella, V. 2006, ApJ, 647, L49

Mennella, V. 2008, ApJ, 684, L25

Mennella, V., Brucato, J. R., Colangeli, L., \& Palumbo, P. 1999, ApJ, 524, L71

Mennella, V., Muñoz Caro, G. M., Ruiterkamp, R., et al. 2001, A\&A, 367, 355

Mennella, V., Brucato, J. R., Colangeli, L., \& Palumbo, P. 2002, ApJ, 569, 531

Mennella, V., Baratta, G. A., Esposito, A., Ferini, G., \& Pendleton, Y. J. 2003, ApJ, 587, 727

Muñoz Caro, G. M., Ruiterkamp, R., Schutte, W. A., Greenberg, J. M., \& Mennella, V. 2001, A\&A, 367, 347

Pendleton, Y. J., \& Allamandola, L. J. 2002, ApJS, 138, 75

Pendleton, Y. J., Sandford, S. A., Allamandola, L. J., Tielens, A. G. G. M., \& Sellgren, K. 1994, ApJ, 437, 683

Perets, H. B., Lederhendler, A., Biham, O., et al. 2007, ApJ, 661, L163

Pety, J., Teyssier, D., Fossé, D., et al. 2005, A\&A, 435, 885

Pino, T., Dartois, E., Cao, A.-T., et al. 2008, A\&A, 490, 665

Pirali, O., Vervloet, M., Dahl, J. E., et al. 2007, ApJ, 661, 919

Pirronello, V., Biham, O., Liu, C., Shen, L., \& Vidali, G. 1997, ApJ, 483, L131

Richter, P., Wakker, B. P., Savage, B. D., \& Sembach, K. R. 2003, ApJ, 586, 230

Risaliti, G., Maiolino, R., Marconi, A., et al. 2006, MNRAS, 365, 303

Sandford, S. A., Allamandola, L. J., Tielens, A. G. G. M., et al. 1991, ApJ, 371, 607

Sandford, S. A., Pendleton, Y. J., \& Allamandola, L. J. 1995, ApJ, 440, 697

Schnaiter, M., Mutschke, H., Dorschner, J., Henning, T., \& Salama, F. 1998, ApJ, 498, 486

Skurat, V. 2003, Nucl. Instrum. Meth. Phys. Res. B, 208, 27

Sloan, G. C., Jura, M., Duley, W. W., et al. 2007, ApJ, 664, 1144

Tielens, A. G. G. M., Wooden, D. H., Allamandola, L. J., Bregman, J., \& Witteborn, F. C. 1996, ApJ, 461, 210

Truica-Marasescu, F.-E., \& Wertheimer, M. R. 2005, Macromol. Chem. Phys., 206, 744

Tyrode, E., \& Hedberg, J. 2011, J. Phys. Chem. C, 116, 1080

Van Dishoeck, E. F., Jonkheid, B., \& van Hemert, M. C. 2006, Faraday Discussions, 133, 231

Vidali, G., Li, L., Roser, J. E., \& Badman, R. 2009, Adv. Space Res., 43, 1291

Wakker, B. P. 2006, ApJS, 163, 282 